\title{
New Backward Recurrences for Bessel Functions
}

\author{
By Henry C. Thacher, Jr.*
}

\begin{abstract}
The recurrences for the coefficients of appropriate power series may be used with the Miller algorithm to evaluate $J_{\nu}(x)\left(|x|\right.$ small), $e^{x} K_{\nu}(x)(\operatorname{Re} x>0$, $|x|$ large), and the modulus and phase of $H_{\nu}^{(1)}(x)(\operatorname{Re} x>0,|x|$ large). The first converges slightly faster than the power series or the classical recurrence, but requires more arithmetic; the last three give both better ultimate precision and faster convergence than the corresponding asymptotic series. The analysis also leads to a formal continued fraction for $K_{\nu+1}(x) / K_{\nu}(x)$ the convergence of which increases with $|x|$. The procedures were tested numerically both for integer and fractional values of $\nu$, and for real and complex $x$.
\end{abstract}

J. C. P. Miller [1] was the first modern worker to apply backward recurrence for evaluating sequences of functions $\left\{f_{k}\right\}$ when the recurrence connecting successive members was unstable for increasing $k$. He evaluated the modified Bessel functions $I_{k}(x)$ by assigning values $F_{N}^{N}=1, F_{N+1}^{N}=0$, and used the recurrence to compute $F_{N-1}^{N}, F_{N-2}^{N}, \ldots, F_{0}^{N}$. For $N>>k$, the $F_{k}^{N}$ approached proportionality to $I_{k}(x)$, and the proportionality constant $C^{N}$ could be evaluated using a generating function. Since 1952, the method has been applied to many families of functions, and the algorithm has been subjected to intensive analysis and refinement. Relatively recent surveys of the status of the method have been provided by Gautschi [2], [3], [4] .

To use conventional backward recurrence methods, one needs a recurrence connecting successive elements of the sequence and, if the recurrence is homogeneous, some normalizing relation such as a generating function or a single function value. The algorithm must also, of course, converge, in the sense that the estimates for a particular element, $f_{k}^{N}=F_{k}^{N} / C^{N}$, approach the true value as $N$ increases. In 1972, Thacher [5] pointed out that solution of linear differential equations with rational coefficients by the method of undetermined coefficients is a fruitful source of recurrences (albeit for the Taylor coefficients, instead of for the elements of a family of functions), and that the value of the function at any point within the circle of convergence of the series provides the required normalizing condition. Moreover, the analytic properties of the solutions of the differential equation provide useful clues to the convergence of the backward recurrence algorithm.

Received May 31, 1977; revised March 9, 1978.

AMS (MOS) subject classifications (1970). Primary 33A40, 33-04, 30A22, 65D20.

Key words and phrases. Bessel functions, modified Bessel functions, Airy function, Hankel function, Kelvin function, power series, continued fraction, differential equation, recurrence, Miller algorithm.

*Work supported, in part, by the National Science Foundation under Grant GJ-41328. 
This paper describes the results of applying this approach to a variety of transformations of Bessel's equation. These transformations allow the evaluation of solutions which have not previously been accessible to the backward recurrence method and include hitherto refractory domains of the independent variable.

1. General Approach. To establish notation and to indicate the general procedure to be followed, we begin with a brief outline of the methods for generating and solving the recurrences. Additional details, which are not important for the present application, may be found in [5].

Let $y^{\dagger}(x)$, the function to be evaluated, satisfy a differential equation which may be transformed to

$$
L w \equiv \sum_{i=0}^{n} \sum_{j=0}^{m_{i}} p_{i j} t^{j} \frac{d^{i} w(t)}{d t^{i}}=\sum_{j=0}^{\infty} h_{j} t^{j} \equiv h(t) .
$$

The changes of dependent variable, $W(x)=F(x, y)=w(t)$, and of independent variable, $x=x(t)$, are chosen to secure the following properties:

a. The origin is an ordinary point of (1-1). Thus, $p_{n 0} \neq 0$, and $h(t)$ is holomorphic for $|t|<r^{\dagger}$.

b. $w^{\dagger}(t)$, the transform of $y^{\dagger}(x)$ is holomorphic in the disk $|t|<r^{\dagger}$, with $r^{\dagger} \geqslant 1$.

c. The value of $w^{\dagger}(1)$ is known, and satisfies $0<\left|w^{\dagger}(1)\right|<\infty$.

Under these circumstances, each solution of (1-1) may be expanded as a series in powers of $t$ with a nonzero radius of convergence

$$
w(t)=\sum_{k=0}^{\infty} \omega_{k} t^{k}, \quad|t|<r, r>0 .
$$

Substituting this expression into the differential equation and collecting the coefficients of equal powers of $t$ leads to a system of equations for the $\omega_{k}$

$$
\sum_{\lambda=0}^{\nu} \Omega_{\lambda}(k) \omega_{\lambda+k}=h_{k} \quad(k=0,1,2, \ldots),
$$

where the coefficients $\Omega_{\lambda}(k)$ are determined by the differential equation.

Although, as pointed out in [5], $\nu$ may be greater than $n$, this is not the case for the equations we consider here, and the coefficients for a set of $n+1(n$ if $h(t) \equiv 0)$ independent solutions of (1-1) form a complete basis for solutions of (1-3). Further, the known value,

$$
w^{\dagger}(1)=\sum_{k=0}^{\infty} \omega_{k}^{\dagger}
$$

gives us an additional condition which may be used for normalization if (1-1) is homogeneous and is otherwise available as a check on convergence.

Although it would be possible to investigate the convergence of the backward recurrence algorithm directly by considering the difference equation (1-3), the relation to the differential equation (1-1) suggests an alternative approach, connecting convergence with the singularities of solutions of (1-1). These, of course, can occur only at the 
singularities of $h(t)$ and at the zeros of the coefficient of $d^{n} w(t) / d t^{n}$. In the case of Bessel functions, they are well known from the properties of the functions.

Letting $w^{\dagger}(t)$ be the desired solution of (1-1) and $w(t)$ be any other distinct solution, the following conjectures have proved useful in predicting convergence of backward recurrence using (1-3) to the coefficients $\left\{\omega_{k}^{\dagger}\right\}$ :

Conjecture 1. A necessary condition for convergence to $\left\{\omega_{k}^{\dagger}\right\}$ is that every other solution $w(t)$ have at least one singularity in the disk $|t| \leqslant r^{\dagger}$.

Conjecture 2. A sufficient condition for convergence is that all the singularities of all the other solutions in a fundamental set including $w^{\dagger}(t)$ lie in the disk $|t|<r^{\dagger}$.

These conjectures leave undetermined the behavior when one of the solutions, $w(t)$, in the fundamental set has a singularity on the circle $|t|=r^{\dagger}$. If this singularity does not coincide with one of the singularities of $w^{\dagger}(t)$, the problem may be resolved by a small shift of origin for $t$. The following conjectures appear to apply when $w^{\dagger}(t)$ and $w(t)$ have a common singular point at $t^{\dagger}=r^{\dagger} e^{i \theta \dagger}$, and no other singularities in $|t| \leqslant r^{\dagger}$, and if $\lim _{r \rightarrow r} \dagger_{-} w^{\dagger}\left(r e^{i \theta \dagger}\right)$ exists.

Conjecture 3. Convergence to $\left\{\omega_{k}^{\dagger}\right\}$ will occur if $w\left(r e^{i \theta \dagger}\right)$ is unbounded as $r \rightarrow r^{\dagger}-$.

Conjecture 4. Convergence to $\left\{\omega_{k}^{\dagger}\right\}$ will occur if $w\left(r e^{i \theta \dagger}\right)$ has an unbounded number of oscillations as $r \rightarrow r^{\dagger}-$.

The principal value of these conjectures is in suggesting appropriate transformations of the original differential equation to secure convergence to the power series coefficients for the desired solution. As for most finite length computations, convergence is neither necessary nor sufficient for utility.

In contrast to the more familiar recurrences, which generate the sequence of members of the family of functions, the recurrence (1-3) yields the sequence of Taylor coefficients. Although the sequences may often be converted into one another, using standard relations for derivatives, the Taylor coefficients themselves have many applications. They may be used to evaluate the function and its derivatives at any point within the circle of convergence; they may be rearranged to a Chebyshev series, providing rapid convergence on the interval $-1 \leqslant t \leqslant 1$; function and derivative values may be used in iterations for finding zeros, and so on. We will emphasize evaluating the function.

The simplest function value to compute is $w^{\dagger}(0)=\omega_{0}^{\dagger}$. However, the values

$$
w^{\dagger}(-1)=\sum_{k=0}^{\infty}(-1)^{k} \omega_{k}^{\dagger}
$$

and

$$
w^{\dagger}( \pm i)=\sum_{k=0}^{\infty}( \pm 1)^{k} i^{k} \omega_{k}^{\dagger}=\sum_{j=0}^{\infty}(-1)^{j} \omega_{2 j}^{\dagger} \pm i \sum_{j=0}^{\infty}(-1)^{j} \omega_{2 j+1}^{\dagger}
$$

may be accumulated without multiplications in parallel with the backward recurrence. It may, thus, be advantageous to choose the origin so that the value of $x$ for which the function is desired corresponds to one of these values of $t$. 
Considerable attention has been devoted to refining algorithms for the backward recurrence approach, particularly for three-term recurrences. Most of this activity has been reviewed in [2], [3], [4]. Although a careful choice of algorithm would be essential for the construction of an acceptable item of software, our computations had a much less ambitious goal, that of exploring the domains of effective convergence of the various recurrences. The original Miller algorithm is quite adequate for this task.

Denoting the approximate normalizing factor and solution for a particular starting value $N$ by $C^{N}$ and $\left\{\omega_{k}^{N}\right\}$, respectively, our computational procedure was as follows: Set $C^{N} \omega_{N}^{N}=1, C^{N} \omega_{N+1}^{N}=C^{N} \omega_{N+2}^{N}=\cdots=0$. For $k=N-1$, $N-2, \ldots, 0$, compute $C^{N} \omega_{k}^{N}$ using (1-3) and accumulate $\Sigma_{j=k}^{N} C^{N} \omega_{j}^{N}$ and $\Sigma_{j=k}^{N} C^{N} \omega_{j}^{N} t^{j-k} \quad$ Then, let

$$
C^{N}=\sum_{j=0}^{N} C^{N} \omega_{j}^{N} / w^{\dagger}(1) ; \quad w_{N}^{\dagger}(t)=w^{\dagger}(1) \sum_{j=0}^{N} C^{N} \omega_{j}^{N} t^{j} / \sum_{j=0}^{N} C^{N} \omega_{j}^{N}
$$

To verify convergence, the recurrence was repeated with $N$ increased by 5 until $\left|\left[w_{N}^{\dagger}(t)-w_{N-5}^{\dagger}(t)\right] / w_{N}^{\dagger}(t)\right|$ was less than some tolerance $\epsilon$, close to machine accuracy. This value of $N$ was denoted by $N^{*}$, and $w_{N^{*}}^{\dagger}(t)$ was chosen as the true value for subsequent estimates of the rate of convergence. When tabulated values were available from independent calculations, this assumption was validated by comparison, but the precision of our values was usually greater than the published values.

The recurrence was then repeated with $N=1(1) N^{*}$ to determine the rate of convergence for smaller values of $N$. The errors were generally expressed as relative precision indexes

$$
P=-\log _{10}\left|\frac{w_{N}^{\dagger}(t)-w_{N^{*}}^{\dagger}(t)}{w_{N^{*}}^{\dagger}(t)}\right|
$$

approximately the number of correct decimal digits produced.

2. Bessel Functions of the First Kind; Small $|x|$. We turn, now, to applications of our approach to Bessel functions. Most of the properties of these functions which we will need are summarized in Chapters 9 [6] and 10 [7] of AMS 55, the notation of which we will follow as closely as possible. Additional useful information about the asymptotic properties which we shall use may be found in [8].

Bessel functions are solutions of Bessel's differential equation

$$
x^{2} y^{\prime \prime}(x)+x y^{\prime}(x)+\left(x^{2}-v^{2}\right) y(x)=0,
$$

where $\nu$ is the order. This equation has a regular singular point at the origin, and an irregular singular point at $\infty$. One solution, the Bessel function of the first kind of order $\nu, J_{\nu}(x)$ has the power series expansion

$$
J_{\nu}(x)=\left(\frac{x}{2}\right)^{\nu} \sum_{k=0}^{\infty} \frac{\left(-x^{2} / 4\right)^{k}}{\Gamma(k+1) \Gamma(k+\nu+1)}, \quad|x|<\infty .
$$


A second solution may be expressed by

$$
Y_{\nu}(x)=\frac{\cos \pi \nu J_{\nu}(x)-J_{-\nu}(x)}{\sin \pi \nu},
$$

when $\nu$ is noninteger, and by the limiting value as $\nu$ approaches an integer value. As $x \rightarrow 0, Y_{0}(x) \sim 2 \ln x / \pi$, while $Y_{\nu}(x) \sim-\Gamma(\nu)(2 / x)^{\nu} / \pi$ when $\operatorname{Re} \nu>0$. Thus, $Y_{\nu}(x)$ has a singularity at the origin.

These facts suggest introducing the new dependent variable $W_{\nu}(x)$ defined by

$$
y(x)=\left(\frac{x}{2}\right)^{\nu} w_{\nu}(x)
$$

The auxiliary function corresponding to $J_{\nu}(x)$ is then an even entire function, taking on the value $1 / \Gamma(\nu+1)$ at the origin, while the function corresponding to $Y_{\nu}(x)$ is of order $(2 / x)^{2 \nu}$ as $x \rightarrow 0$ for $\operatorname{Re} \nu>0$, and of order $\ln (x / 2)$ for $\nu=0$.

The symmetry of the auxiliary function corresponding to $J_{\nu}(x)$, and the fact that its value is known at the origin suggests introducing the new independent variable $t$ by

$$
x(t)=z \sqrt{1-t}, \quad t(x)=1-(x / z)^{2},
$$

where the parameter $z$ may be chosen freely, although, as we shall see, increasing $|z|$ decreases the rate of convergence of the Miller algorithm.

Letting $w(t)=W(x[t])$, and introducing the new variables into Bessel's equation leads to

$$
(1-t) w^{\prime \prime}(t)-(\nu+1) w^{\prime}(t)+(z / 2)^{2} w(t)=0,
$$

which has an ordinary point at the origin, and singular points at $t=1$ and $t=\infty$.

If we write

$$
w^{\dagger}(t)=\left(\frac{2}{z \sqrt{1-t}}\right)^{\nu} J_{\nu}(z \sqrt{1-t})
$$

for the solution corresponding to $J_{\nu}(x)$, and

$$
\widetilde{w}(t)=\left(\frac{2}{z \sqrt{1-t}}\right)^{\nu} Y_{\nu}(z \sqrt{1-t})
$$

for the solution corresponding to $Y_{\nu}(x)$, we observe that $w^{\dagger}(t)$ is an entire function of $t$, with $w^{\dagger}(1)=1 / \Gamma(\nu+1)$, while $\tilde{w}(t)$ is holomorphic for $|t|<1$, but has singularities at $t=1$ and $t=\infty$. Conjecture 1, thus, implies that if the Miller algorithm converges, it will converge to the coefficients for $w^{\dagger}(t)$.

Introducing the power series (1-2), with $r=1$, into (2-6) leads to the recurrence

$$
\omega_{k-1}=\left(\frac{2}{z}\right)^{2} k\left[(k+v) \omega_{k}-(k+1) \omega_{k+1}\right]
$$


with the additional condition

$$
w^{\dagger}(1)=\sum_{k=0}^{\infty} \omega_{k}^{\dagger}=1 / \Gamma(\nu+1)
$$

As indicated in the last section, an appropriate choice of $z$ can simplify the calculations significantly, and may also improve the rate of convergence. If $z$ is real, or pure imaginary, (2-9) and (2-10) include only real quantities. Thus, both $J_{\nu}(x)$ and $I_{\nu}(x)$ (the modified Bessel function) may be evaluated for real $x$ without resorting to complex arithmetic. The evaluation of $w^{\dagger}(t)$ is, of course, simplest for $t=0$, i.e. for $z^{2}=x^{2}$. However, the rate of convergence of the Miller algorithm decreases as $|z|$ increases, so that for real or pure imaginary $x$ the choice $z^{2}=x^{2} / 2$, and (1-5) may be advantageous.

The recurrence (2-9) was tested numerically using approximately $16 \mathrm{~S}$ floating point arithmetic for real $x$ and $v$ as high as 16 , with $z=x$ and $z=x / \sqrt{2}$. The results, typical examples of which are shown in Table 2-1, lead to the following general observations: The recurrence does, in fact, converge, to correct values, even for $x$ as large as 16. The precision for a given starting index increases with $\nu$, and decreases with $|x|$. Precision is somewhat higher with $z=x / \sqrt{2}$ than with $z=x$, but the improvement may not be sufficient to justify the extra computation required in using (1-5).

TABLE 2-1

Index of precision for $J_{\nu}(x)$. Miller algorithm on Eq. (2-9).

\begin{tabular}{|c|c|c|c|c|c|c|c|c|}
\hline$v$ & 0 & 0 & 0 & 0 & 0 & 8 & 0 & 0 \\
\hline$x$ & 1 & 1 & 2 & 8 & 8 & 8 & 16 & 16 \\
\hline 2 & $1 / \sqrt{2}$ & 1 & 2 & $4 \sqrt{2}$ & 8 & 8 & $8 \sqrt{2}$ & 16 \\
\hline $\mathbf{N}$ & \multicolumn{7}{|c|}{ Index of Precision } & \\
\hline 2 & 3.32 & 2.60 & 0.56 & 0.41 & -0.19 & $0.3 i$ & -0.73 & 0.08 \\
\hline 4 & 7.43 & 6.06 & 2.74 & 0.41 & -0.01 & 1.44 & -0.47 & -0.01 \\
\hline 6 & 12.30 & 10.31 & 5.76 & 0.98 & 0.48 & 2.88 & -0.34 & 0.00 \\
\hline 8 & 16.74 & 15.06 & 9.32 & 2.37 & 1.79 & 4.68 & -0.23 & 0.00 \\
\hline 10 & & & 13.29 & 4.35 & 2.39 & 6.76 & -0.06 & -0.01 \\
\hline 12 & & & 15.61 & 6.72 & 3.77 & 9.10 & 0.44 & -0.01 \\
\hline 14 & & & & 9.40 & 5.66 & 11.66 & 1.58 & 0.01 \\
\hline 16 & & & & 12.33 & 7.87 & 14.37 & 3.17 & 0.15 \\
\hline 18 & & & & 15.79 & 10.34 & 15.91 & 5.03 & 0.76 \\
\hline 20 & & & & & 13.03 & & 7.11 & 1.96 \\
\hline 22 & & & & & 16.09 & & 9.38 & 3.49 \\
\hline 24 & & & & & & & 11.82 & 5.21 \\
\hline 26 & & & & & & & $\infty$ & 7.12 \\
\hline 28 & & & & & & & & 9.17 \\
\hline 30 & & & & & & & & 11.36 \\
\hline
\end{tabular}


Two other methods of evaluating $J_{\nu}(x)$ for relatively small $|x|$ also deserve consideration: backward application of the classical recurrence

$$
J_{\nu+k-1}(x)=\frac{2}{x}(\nu+k) J_{\nu+k}(x)-J_{\nu+k+1}(x)
$$

and the classical power series (2-2). Comparative evaluations of $J_{0}(x)$ by all three methods showed that for fixed $N,(2-9)$ with $z=x / \sqrt{2}$ was more precise than any of the other methods, while (2-11) was uniformly the worst, except for $x \geqslant 8$, where the power series was inadequate. Even with $z=x,(2-9)$ was superior to (2-11) and to the power series for $x \geqslant 4$. The starting point necessary to attain a specified precision is not, however, the sole criterion for the choice of algorithm, and it should be observed that the amount of arithmetic for each application of (2-9) is significantly greater than for (2-11) or (2-2). A final selection would require a detailed analysis of specific programs for the various domains.

3. Modified Bessel Functions of the Second Kind, Large $x$. Changing the independent variable in Bessel's equation to $i x$ leads to the modified Bessel equation

$$
x^{2} y^{\prime \prime}(x)+x y^{\prime}(x)-\left(x^{2}+v^{2}\right) y(x)=0,
$$

with independent solutions

and

$$
I_{\nu}(x)= \begin{cases}e^{-\nu \pi i / 2} J_{\nu}\left(x e^{\pi i / 2}\right) & (-\pi<\arg x \leqslant \pi / 2), \\ e^{3 \nu \pi i / 2} J_{\nu}\left(x e^{-3 \pi i / 2}\right) & (\pi / 2<\arg x \leqslant \pi),\end{cases}
$$

$$
K_{\nu}(x)=\pi\left[I_{-\nu}(x)-I_{\nu}(x)\right] / 2 \sin \nu \pi
$$

with the limit being taken, as usual, when $\nu$ is an integer.

For $|x|$ small, $I_{\nu}(x)$ can be evaluated by the methods of the last section. For large $|x|$, we may now use the backward recurrence method to evaluate $K_{\nu}(x)$. In the neighborhood of the irregular singular point at $\infty$, the solutions have the asymptotic expansions

$$
\begin{aligned}
& I_{\nu}(x) \sim \frac{e^{x}}{\sqrt{2 \pi x}}\left\{1-\frac{4 \nu^{2}-1}{8 x}+\frac{\left(4 \nu^{2}-1\right)\left(4 \nu^{2}-9\right)}{2 !(8 x)^{2}}-\cdots\right\} \quad(|\arg x|<\pi / 2), \\
& K_{\nu}(x) \sim \sqrt{\frac{\pi}{2 x}} e^{-x}\left\{1+\frac{4 \nu^{2}-1}{8 x}+\frac{\left(4 \nu^{2}-1\right)\left(4 \nu^{2}-9\right)}{2 !(8 x)^{2}}+\cdots\right\} \\
& \quad(|\arg x|<3 \pi / 2)
\end{aligned}
$$

Since $K_{\nu}(x)$ is recessive at $\infty$, we hope that it will be computable by our procedure. To obtain a function with a nonzero value at $\infty$ we introduce the new dependent variable $W(x)$ by setting

$$
y(x)=\sqrt{\frac{\pi}{2 x}} e^{-x} W(x),
$$


so that the differential equation becomes

$$
x^{2} W^{\prime \prime}(x)-2 x^{2} W^{\prime}(x)-\frac{4 v^{2}-1}{4} W(x)=0
$$

with the solution corresponding to $K_{\nu}(x)$ approaching 1 as $x \rightarrow \infty$ in $|\arg x|<$ $3 \pi / 2$, while the solution corresponding to $I_{\nu}(x)$ approaches $e^{2 x} / \pi$.

Since the differential equation is no longer invariant under a change of sign of $x$, we use the simple change of variable

$$
x(t)=z /(1-t), \quad t(x)=1-(z / x),
$$

to map the point $x=\infty$ onto $t=1$. Letting $w(t)=W(x[t])$, our differential equation becomes

$$
(1-t)^{2} w^{\prime \prime}(t)-2(1+z-t) w^{\prime}(t)-\frac{4 \nu^{2}-1}{4} w(t)=0
$$

with the boundary condition $w(1)=1$ corresponding to the solution for $K_{\nu}(x)$.

Again, the origin is an ordinary point, so that the power series

$$
w(t)=\sum_{k=0}^{\infty} \omega_{k} t^{k}
$$

converges for $|t|<1$. The method of undetermined coefficients leads to a recurrence for the $\omega_{k}$ which can be written in the form

$$
\omega_{k-1}=\frac{2(k+z) \omega_{k}-(k+1) \omega_{k+1}}{k-1-\left(4 \nu^{2}-1\right) / 4 k} .
$$

Since the solution corresponding to $\sqrt{2 x / \pi} e^{x} K_{\nu}(x)$ approaches 1 as $t \rightarrow 1$, while the solution corresponding to $\sqrt{2 x / \pi} e^{x} I_{\nu}(x)$ is unbounded, Conjecture 3 suggests that the backward recurrence solution of (3-11) will converge to $\left\{\omega_{k}^{\dagger}\right\}$ with

$$
w^{\dagger}(1)=\sum_{k=0}^{\infty} \omega_{k}^{\dagger}=1, \quad w^{\dagger}(t)=\sum_{k=0}^{\infty} \omega_{k}^{\dagger} t^{k}=\sqrt{\frac{2 z}{\pi(1-t)}} e^{z /(1-t)} K_{\nu}\left(\frac{z}{1-t}\right)
$$

In particular, for $t=0$,

$$
w^{\dagger}(0)=\omega_{0}^{\dagger}=\sqrt{2 z / \pi} e^{z} K_{\nu}(z)
$$

and for $t=-1$,

$$
w^{\dagger}(-1)=\sum_{k=0}^{\infty} \omega_{k}^{\dagger}(-1)^{k}=\sqrt{z / \pi} e^{z / 2} K_{\nu}(z / 2)
$$

requiring no multiplications in summing the series.

Like all three-term homogeneous recurrences, (3-11) has an associated sequence of formal continued fractions. These may be written in $J$-fraction form

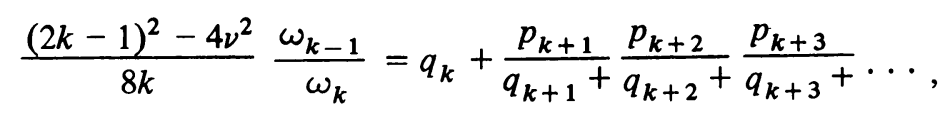


where

$$
p_{j}=\left[4 \nu^{2}-(2 j-1)^{2}\right] / 16, \quad q_{j}=j+z
$$

and $k=1,2,3, \ldots$

By Pincherle's theorem, our conjecture on the convergence of the backward recurrence algorithm implies the convergence of this fraction to the ratio of consecutive elements of the minimal solution $\left\{\omega_{k}^{\dagger}\right\}$.

For $k=1,(3-15)$ yields a continued fraction for the logarithmic derivative of $w(t)$ at $t=0$

$$
\frac{w^{\prime}(0)}{w(0)}=\frac{\omega_{1}}{\omega_{0}}=\frac{\left(1-4 v^{2}\right) / 8}{q_{1}+} \frac{p_{2}}{q_{2}+} \frac{p_{3}}{q_{3}+\ldots} .
$$

Returning to the modified Bessel functions, we have, from (3-6),

$$
\ln K_{\nu}(z)=\ln \sqrt{\frac{\pi}{2}}-\frac{1}{2} \ln z-z+\ln W(z)
$$

so that

$$
-\frac{K_{\nu}^{\prime}(z)}{K_{\nu}(z)}=\frac{1}{2 z}+1-\frac{W^{\prime}(z)}{W(z)}=1+\frac{1}{2 z}-\frac{1}{z} \frac{w^{\prime}(0)}{w(0)} .
$$

Using the recurrence

$$
K_{\nu}^{\prime}(z)=-K_{\nu+1}(z)+\frac{v}{z} K_{\nu}(z)
$$

we obtain

$$
\frac{K_{\nu+1}(z)}{K_{\nu}(z)}=\frac{\nu}{z}-\frac{K_{\nu}^{\prime}(z)}{K_{\nu}(z)}=1+\frac{2 \nu+1}{2 z}+\frac{\left(4 \nu^{2}-1\right) / 8 z}{1+z+} \frac{\left(4 \nu^{2}-9\right) / 16}{2+z+\cdots} .
$$

This fraction agrees with the odd part of one of Hitotumatu's [9, Eq. 4.7] convergent continued fractions for $K_{\nu+1}(x) / K_{\nu}(x)$. Thus, the convergence of the Miller algorithm follows from Pincherle's theorem. In contrast to the direct use of the continued fraction, the backward recurrence provides a simple normalization procedure.

The method is not, of course, restricted to integer orders, or to real arguments. For $\nu=(2 n-1) / 2$, the denominator in (3-11) vanishes for $k=n$, but this merely reflects the fact that modified Bessel functions of half-integer order have finite expansions in powers of $1 / x$. Exact results will be obtained by starting the recurrence with $\omega_{n}=0, \omega_{n-1}=1$.

Among the modified Bessel functions of fractional order which are accessible by this technique, the Airy functions,

$$
\mathrm{Ai}(s)=\frac{1}{\pi} \sqrt{\frac{s}{3}} K_{1 / 3}\left(\frac{2 s^{3 / 2}}{3}\right), \quad \mathrm{Ai}^{\prime}(s)=\frac{-s}{\pi \sqrt{3}} K_{2 / 3}\left(\frac{2 s^{3 / 2}}{3}\right)
$$

have attracted particular interest. Since $\mathrm{Ai}(s)$ is the recessive solution of Airy's equation as $|s| \rightarrow \infty$ within the sector $|\arg s|<\pi / 3$, backward recurrence is effective within this sector. 
In the complex plane, the Kelvin functions of the third kind,

$$
\operatorname{ker}_{\nu}(s)+i \operatorname{kei}_{\nu}(s) \equiv e^{-\nu \pi i / 2} K_{\nu}\left(s e^{i \pi / 4}\right)
$$

have found considerable application. They are accessible by backward recurrence using the representation

$$
\operatorname{ker}_{\nu}(s)+i \operatorname{kei}_{\nu}(s)=\exp \left\{-\left(\frac{4 \nu+1}{8} \pi+\frac{s}{\sqrt{2}}\right)^{i}\right\} \sqrt{\frac{\pi}{2 s}} e^{-s / \sqrt{2}} W_{\nu}\left(s e^{i \pi / 4}\right)
$$

For $-\pi<\arg s \leqslant \pi / 2, K_{\nu}(s)$ can be expressed in terms of the Bessel function of the third kind (Hankel functions)

$$
K_{\nu}(s)=(i \pi / 2) e^{i \nu \pi / 2} H_{\nu}^{(1)}\left(s e^{i \pi / 2}\right)
$$

where $H_{\nu}^{(1)}(x) \equiv J_{\nu}(x)+i Y_{\nu}(x)$. Accordingly,

$$
H_{\nu}^{(1)}(x)=\frac{2}{\pi i} e^{-i \nu \pi / 2} K_{\nu}\left(x e^{-i \pi / 2}\right)=\sqrt{\frac{2}{\pi x}} e^{-i(2 \nu+1) / 4} e^{i x} W_{\nu}\left(x e^{-i \pi / 2}\right)
$$

The real and imaginary parts of $W_{\nu}(-i x)$ can thus be identified with the functions $P(\nu, x)$ and $Q(\nu, x)$ appearing in Hankel's asymptotic representations

$$
\begin{aligned}
& J_{\nu}(x)=\sqrt{\frac{2}{\pi x}}\{P(\nu, x) \cos \chi-Q(\nu, x) \sin \chi\}, \\
& Y_{\nu}(x)=\sqrt{\frac{2}{\pi x}}\{P(\nu, x) \sin \chi+Q(\nu, x) \cos \chi\},
\end{aligned}
$$

where $\chi=x-(2 \nu+1) \pi / 4$.

The procedure was tested by numerical calculations in $16 \mathrm{~S}$ arithmetic for various orders, arguments, and choices for the parameter $z$. The method was validated by comparisons with values of $K_{0}(2), K_{1}(2), \operatorname{ker} 2$ and kei 2 and $J_{0}(2)$ and $Y_{0}(2)$ tabulated in AMS 55 [6]. Agreement to the accuracy of the tabulated values was obtained in all four test cases.

For other cases, reference values and precision indexes were computed by the method of Section 1, with $\epsilon=10^{-15}$. Typical results are given in Table 3-1. For comparison, the maximum precision obtainable by the asymptotic series [6, Eq. 9.7.2] for $x=16$ is 14.74 and requires 33 terms.

Although the whole domain of $x, \nu$, and $z$ was not explored, the following semiquantitative generalizations appear useful:

a. For fixed $z$ and $\nu$, the index of precision, $P$, is effectively independent of $t$ for $|t| \leqslant 1$.

b. For fixed $z$ and $\nu$, with $|\nu|<|z|$, the index of precision for varying starting points, $N$, may be represented by an expression of the form 


$$
P=\alpha N^{\beta}
$$

with $\beta \cong .5$ for $\nu \leqslant 1$, increasing somewhat with $\nu$. For $\nu>|z|$, convergence is unacceptably slow.

\section{TABLE 3-1}

Precision indexes for $\sqrt{\frac{2 x}{\pi}} e^{x} K_{\nu}(x)$ by backward recurrence.

\begin{tabular}{|c|c|c|c|c|c|c|c|}
\hline$|x|$ & 1.0 & 2.0 & 16.0 & 2.0 & 1.0 & $\sqrt{2}$ & 2.0 \\
\hline $\arg x$ & 0. & 0 & 0 & 0 & $-\pi / 2$ & $\pi / 4$ & 0 \\
\hline$|z|$ & 2.0 & 2.0 & 32.0 & 4.0 & 2.0 & $2 \sqrt{2}$ & 4.0 \\
\hline $\arg 2$ & 0 & 0 & 0 & 0 & $-\pi / 2$ & $\pi / 4$ & 0 \\
\hline v & 0 & 0 & 0 & $2 / 3$ & 0 & 0 & 2 \\
\hline $\mathbf{N}$ & \multicolumn{6}{|c|}{ Precision Indexes } & \\
\hline 1 & 2.12 & 1.96 & 5.15 & 2.94 & 1.84 & 2.71 & 1.40 \\
\hline 2 & 2.41 & 2.52 & 5.42 & 3.30 & 2.20 & 3.02 & 2.38 \\
\hline 3 & 3.09 & 2.99 & 7.76 & 4.27 & 2.65 & 3.94 & 3.37 \\
\hline 4 & 3.34 & 3.41 & 8.05 & 4.57 & 2.92 & 4.22 & 3.89 \\
\hline 5 & 3.86 & 3.79 & 10.04 & 5.32 & 3.25 & 4.93 & 4.59 \\
\hline 6 & 4.09 & 4.14 & 10.33 & 5.61 & 3.48 & 5.19 & 5.01 \\
\hline 7 & 4.52 & 4.47 & 12.09 & 6.23 & 3.75 & 5.78 & 5.59 \\
\hline 8 & 4.73 & 4.77 & 12.38 & 6.49 & 3.95 & 6.03 & 5.95 \\
\hline 9 & 5.11 & 5.06 & 13.98 & 7.04 & 4.18 & 6.53 & 6.45 \\
\hline 10 & 5.30 & 5.34 & 14.27 & 7.29 & 4.37 & 6.77 & 6.78 \\
\hline 11 & 5.64 & 5.60 & 15.85 & 7.77 & 4.58 & 7.22 & 7.22 \\
\hline 12 & 5.82 & 5.85 & 16.16 & 8.01 & 4.75 & 7.44 & 7.52 \\
\hline 13 & 6.12 & 6.09 & 16.55 & 8.45 & 4.94 & 7.85 & 7.92 \\
\hline 14 & 6.30 & 6.32 & & 8.68 & 5.10 & 8.07 & 8.20 \\
\hline 15 & 6.58 & 6.55 & & 9.09 & 5.27 & 8.44 & 8.57 \\
\hline 16 & 6.74 & 6.77 & & 9.30 & 5.42 & 8.65 & 8.84 \\
\hline 17 & 7.01 & 6.98 & & 9.68 & 5.58 & 9.00 & 9.19 \\
\hline 18 & 7.16 & 7.19 & & 9.89 & 5.73 & 9.19 & 9.44 \\
\hline 19 & 7.41 & 7.39 & & 10.24 & 5.88 & 9.52 & 9.76 \\
\hline 20 & 7.56 & 7.58 & & 10.44 & 6.01 & 9.71 & 10.00 \\
\hline 21 & 7.80 & 7.77 & & 10.78 & 6.16 & 10.02 & 10.31 \\
\hline 22 & 7.94 & 7.96 & & 10.97 & 6.29 & 10.21 & 10.54 \\
\hline 23 & 8.16 & 8.14 & & 11.29 & 6.43 & 10.50 & 10.83 \\
\hline 24 & 8.30 & 8.32 & & 11.48 & 6.55 & 10.68 & 11.06 \\
\hline 25 & 8.52 & 8.50 & & 11.79 & 6.68 & 10.96 & 11.33 \\
\hline 50 & 12.09 & 12.10 & & & 9.27 & 15.47 & \\
\hline 75 & 15.02 & 15.01 & & & 11.25 & & \\
\hline
\end{tabular}


c. The constant $\alpha$ in (3-29) depends primarily on $|z|$, and varies only slightly with $\arg z$ and $\nu$ for $\nu<|z|$. The dependence may be approximated by

$$
\alpha=1.4|z|^{.33} \text {. }
$$

4. Bessel Function Modulus. As $|x| \rightarrow \infty$ with $x$ near the real axis, both $J_{\nu}(x)$ and $Y_{\nu}(x)$ oscillate with comparable amplitudes and with periods approaching $2 \pi$. This behavior is evident from the Hankel representations (3-27) and (3-28), since the auxiliary functions $P(\nu, x)$ and $Q(\nu, x)$ have the asymptotic series representations

$$
\begin{aligned}
P(\nu, x) \sim & 1-\frac{\left(4 v^{2}-1\right)\left(4 v^{2}-9\right)}{2 !(8 x)^{2}} \\
& +\frac{\left(4 v^{2}-1\right)\left(4 v^{2}-9\right)\left(4 v^{2}-25\right)\left(4 v^{2}-49\right)}{4 !(8 x)^{4}}-\cdots, \\
Q(\nu, x) \sim & \frac{4 v^{2}-1}{8 x}-\frac{\left(4 v^{2}-1\right)\left(4 v^{2}-9\right)\left(4 v^{2}-25\right)}{3 !(8 x)^{3}}+\cdots
\end{aligned}
$$

Even with the convergent recurrence of Section 3 for computing the auxiliary functions, the Hankel form requires two trigonometric functions for either of the Bessel functions, and, more seriously, is subject to cancellation error in the neighborhood of the zeros. Alternate representations valid for large $x$ are, thus, desirable.

The oscillation of $J_{\nu}(x)$ and $Y_{\nu}(x)$ suggests that no power series for either solution of Bessel's equation which is valid as $x \rightarrow \infty$ will have minimal coefficients. If, however, we turn to products of solutions, we find

$$
\begin{aligned}
J_{\nu}^{2}(x) & +Y_{\nu}^{2}(x)=\frac{2}{\pi x}\left\{P^{2}(\nu, x)+Q^{2}(\nu, x)\right\} \sim \frac{2}{\pi x} \\
2 J_{\nu}(x) Y_{\nu}(x) & =\frac{2}{\pi x}\left\{\left[P^{2}(\nu, x)-Q^{2}(\nu, x)\right] \sin 2 \chi+2 P(\nu, x) Q(\nu, x) \cos 2 \chi\right\} \\
& \sim \frac{2 \sin 2 \chi}{\pi x}, \\
J_{\nu}^{2}(x)-Y_{\nu}^{2}(x) & =\frac{2}{\pi x}\left\{\left[P^{2}(\nu, x)-Q^{2}(\nu, x)\right] \cos 2 \chi-2 P(\nu, x) Q(\nu, x) \sin 2 \chi\right\} \\
& \sim \frac{2 \cos 2 \chi}{\pi x},
\end{aligned}
$$

and only the last two oscillate as $x \rightarrow \infty$. Conjecture 4 then suggests that if we can find a differential equation with (4-3), (4-4) and (4-5) as a complete set of independent solutions, backward recurrence on the power series coefficients will converge to the coefficients for $J_{\nu}^{2}(x)+Y_{\nu}^{2}(x)$.

Such a differential equation can be obtained using the result $[10$, p. 298 , Example 10] that if $y_{1}$ and $y_{2}$ are independent solutions of the differential equation 


$$
y^{\prime \prime}+R y^{\prime}+S y=0,
$$

then $y_{1}^{2}, y_{1} y_{2}$, and $y_{2}^{2}$ are independent solutions of

$$
u^{\prime \prime \prime}+3 R u^{\prime \prime}+\left(2 R^{2}+\frac{d R}{d x}+4 S\right) u^{\prime}+\left(4 R S+2 \frac{d S}{d x}\right) u=0 .
$$

Writing Bessel's equation in the form

$$
y^{\prime \prime}(x)+\frac{1}{x} y^{\prime}(x)+\left(1-\frac{v^{2}}{x^{2}}\right) y(x)=0,
$$

we see that the general solution of

$$
u^{\prime \prime \prime}(x)+\frac{3}{x} u^{\prime \prime}(x)+\frac{1-4 v^{2}+4 x^{2}}{x^{2}} u^{\prime}(x)+\frac{4}{x} u(x)=0
$$

can be written as

$$
u(x)=A\left[J_{\nu}^{2}(x)+Y_{\nu}^{2}(x)\right]+2 B J_{\nu}(x) Y_{\nu}(x)+C\left[J_{\nu}^{2}(x)-Y_{\nu}^{2}(x)\right],
$$

where $J_{\nu}^{2}(x)+Y_{\nu}^{2}(x)$ is the squared modulus of the Hankel function, often written as $M_{\nu}^{2}(x)$.

Letting $u(x)=W(x) / x$ leads to the conventional differential equation for the squared modulus of the Hankel function

$$
x^{3} W^{\prime \prime \prime}(x)+\left(1-4 \nu^{2}+4 x^{2}\right) x W^{\prime}(x)+\left(4 \nu^{2}-1\right) W(x)=0
$$

with the three independent solutions

$$
\begin{gathered}
W_{1}(x)=x\left[J_{\nu}^{2}(x)+Y_{\nu}^{2}(x)\right], \quad W_{2}(x)=2 x J_{\nu}(x) Y_{\nu}(x), \\
W_{3}(x)=x\left[J_{\nu}^{2}(x)-Y_{\nu}^{2}(x)\right] .
\end{gathered}
$$

From our outline of the asymptotic properties, we see that $W_{1}(x)$ is a symmetric function of $x$, and that

$$
\lim _{x \rightarrow \infty} W_{1}(x)=\limsup _{x \rightarrow \infty} W_{2}(x)=\limsup _{x \rightarrow \infty} W_{3}(x)=\frac{2}{\pi},
$$

where both $W_{2}(x)$ and $W_{3}(x)$ oscillate with periods approaching $\pi$ as $x \rightarrow \infty$.

In view of the symmetry of $W_{1}(x)$, the change of independent variable

$$
x(t)=z / \sqrt{1-t}, \quad t(x)=1-(z / x)^{2} \quad(\operatorname{Re} x>0, \operatorname{Re} z>0)
$$

is appropriate, letting, as usual, $w(t)=W(x[t])$. With this change of variable, (4-11) becomes

(4-15) $2(1-t)^{3} w^{\prime \prime \prime}(t)-9(1-t)^{2} w^{\prime \prime}(t)+2\left[z^{2}+(3-\lambda)-(3-\lambda) t\right] w^{\prime}(t)+\lambda w(t)=0$

where $4 \lambda=4 \nu^{2}-1$. The boundary condition corresponding to $W(\infty)=2 / \pi$ is $w(1)=2 / \pi$. 
Letting $w(t)=\Sigma \omega_{k} t^{k}$, and substituting in (4-15) leads, after some rearrangement, to

$$
\begin{aligned}
& \sum_{j=1}^{\infty}\left\{2 j(j+1)(j+2) \omega_{j+2}-3 j(j+1)(2 j+1) \omega_{j+1}\right. \\
& \left.\quad+2 j\left(3 j^{2}+z^{2}-\lambda\right) \omega_{j}-\left[2 j^{3}-3 j^{2}+(1-2 \lambda) j+\lambda\right] \omega_{j-1}\right\} t^{j-1}=0
\end{aligned}
$$

so that the coefficients $\omega_{j}$ must satisfy the four term recurrence

$$
\begin{aligned}
\underset{(4-17)}{\omega_{k-1}}=\frac{k}{2 k^{3}-3 k^{2}+(1-2 \lambda) k+\lambda} & \left\{(k+1)\left[2(k+2) \omega_{k+2}-(6 k+3) \omega_{k+1}\right]\right. \\
& \left.+2\left(3 k^{2}+z^{2}-\lambda\right) \omega_{k}\right\}
\end{aligned}
$$

with the normalizing condition

$$
\sum_{k=0}^{\infty} \omega_{k}=\frac{2}{\pi} .
$$

The applicability of the Miller algorithm with (4-17) and (4-18) was verified by numerical calculation with a variety of real orders and arguments. For most of the tests the parameter $z$ was taken equal to $x$, but the choice $z=\sqrt{2 x}$ was also examined (and found to be advantageous), and one test was made with $\nu=0, x=8$ and $z=$ $6+2 i$ to verify the applicability of the algorithm for complex values. Fractional orders caused no difficulty except, of course, for $\nu=(2 m+1) / 2$ where the series terminates. Selected results were found to be in satisfactory agreement with values computed from the tables in AMS 55 [6].

The rate of convergence was explored by calculations similar to those described in Section 3. The data, typical examples of which are shown in Table 4-1, support the following conclusions:

a. The choice $z=\sqrt{2 x}$ gives more rapid convergence than $z=x$.

b. For fixed $z$ and $\nu$, the precision indexes are well represented by an expression of the form (3-29), with $\alpha$ decreasing from 5.33 to 2.02 , and $\beta$ from 0.45 to 0.34 as $z$ decreases from 16 to 2 .

c. The rate of convergence decreases with increasing order. The technique is of little value for $\nu$ significantly greater than $|z|$.

For comparison, the asymptotic series [6, Eq. 9.2.28] requires 17 terms to achieve a maximum precision of 14.45 for $x=16$, and 9 terms give a maximum precision 7.36 for $x=8$.

5. Evaluation of the Phase. The phase, $\theta_{\nu}(x)$, for Bessel functions may be defined by

$$
J_{\nu}(x)=M_{\nu}(x) \cos \theta_{\nu}(x), \quad Y_{\nu}(x)=M_{\nu}(x) \sin \theta_{\nu}(x)
$$

where $M_{\nu}(x)$ is the modulus, which was considered in the last section. 


\section{TABLE 41}

Precision indexes for $x\left[J_{\nu}^{2}(x)+Y_{\nu}^{2}(x)\right]$ by backward recurrence using Eqs. (4-17) and (4-18).

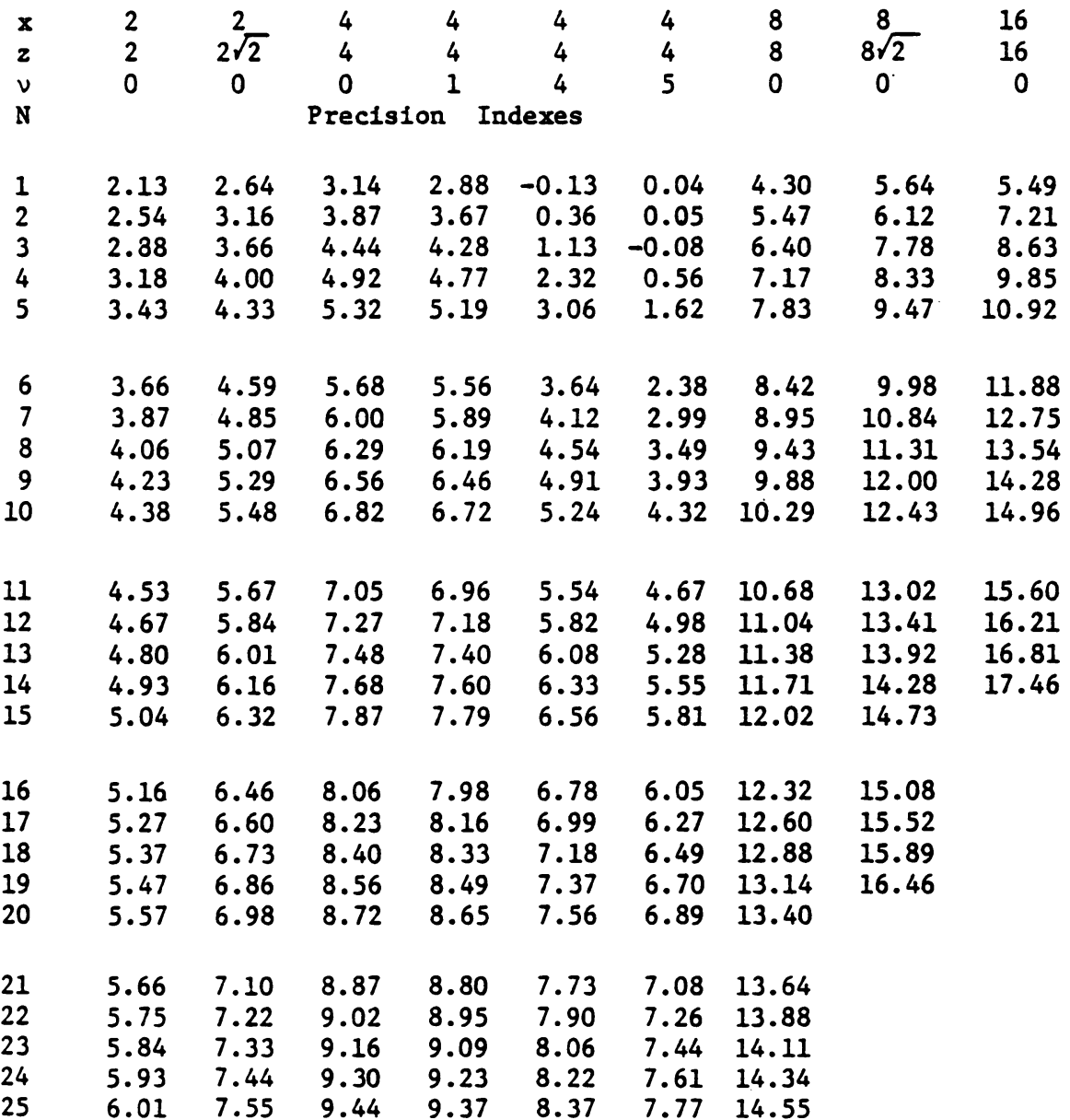

Writing the Wronskian for $J_{\nu}(x)$ and $Y_{\nu}(x)$ in terms of modulus and phase

$$
\left|\begin{array}{ll}
J_{\nu}(x) & Y_{\nu}(x) \\
J_{\nu}^{\prime}(x) & Y_{\nu}^{\prime}(x)
\end{array}\right|
$$

we find

$$
=\left|\begin{array}{ll}
M_{\nu} \cos \theta_{\nu} & M_{\nu} \sin \theta_{\nu} \\
M_{\nu}^{\prime} \cos \theta_{\nu}-M_{\nu} \sin \theta_{\nu} \theta_{\nu}^{\prime} & M_{\nu}^{\prime} \sin \theta_{\nu}+M_{\nu} \cos \theta_{\nu} \theta_{\nu}^{\prime}
\end{array}\right|=\frac{2}{\pi x},
$$

$$
\left|\begin{array}{cc}
M_{\nu} \cos \theta_{\nu} & M_{\nu} \sin \theta_{\nu} \\
-M_{\nu} \sin \theta_{\nu} \theta_{\nu}^{\prime} & M_{\nu} \cos \theta_{\nu} \theta_{\nu}^{\prime}
\end{array}\right|=M_{\nu}^{2} \theta_{\nu}^{\prime}=\frac{2}{\pi x}
$$


leading to the familiar differential equation

$$
x M_{\nu}^{2}(x) \theta_{\nu}^{\prime}(x)=2 / \pi .
$$

As $|x| \rightarrow \infty$, the phase has the asymptotic representation

$$
\theta_{\nu}(x) \sim x-\frac{2 \nu+1}{4} \pi+\frac{4 \nu^{2}-1}{2(4 x)}+\frac{\left(4 \nu^{2}-1\right)\left(4 \nu^{2}-25\right)}{6(4 x)^{3}}+\cdots,
$$

suggesting that we write

$$
\theta_{\nu}(x)=\chi_{\nu}(x)+\frac{D_{\nu}(x)}{x},
$$

where, as in the last section, $\chi_{\nu}(x) \equiv x-(2 \nu+1) \pi / 4$, and the auxiliary function $D_{\nu}(x)$ is an even function of $x$ satisfying

$$
D_{\nu}(x) \sim \frac{4 \nu^{2}-1}{8}, \quad D_{\nu}^{\prime}(x) \sim \frac{-\left(4 \nu^{2}-1\right)\left(4 \nu^{2}-25\right)}{3(4 x)^{3}} .
$$

In terms of the auxiliary functions, $W_{\nu}(x)$ (from the last section) and $D_{\nu}(x)$, we may write the differential equation (5-4) as

$$
W_{\nu}(x)\left[\frac{D_{\nu}^{\prime}(x)}{x}-\frac{D_{\nu}(x)}{x^{2}}+1\right]=\frac{2}{\pi}
$$

Again, changing the independent variable to

$$
t=1-z^{2} / x^{2}
$$

and letting $d(t)=D_{\nu}(x[t])$, we obtain, after simplification

$$
2(1-t) d^{\prime}(t)-d(t)=r(t)
$$

where

$$
r(t)=\frac{[2 / \pi-w(t)] z^{2}}{(1-t) w(t)}=\frac{[w(1)-w(t)] z^{2}}{(1-t) w(t)} .
$$

The singularities of $r(t)$ will fall at $t=1$ (because of the essential singularity of $M_{\nu}^{2}(x)$ at $x=\infty$ ) and at the zeros of $w(t)$ (i.e. at $t=\infty$, and at the values of $t$ corresponding to the complex zeros of $\left.H_{\nu}^{(1)}(x)\right)$. If all of these zeros lie outside the circle $t=1$, we can represent $r(t)$ as a power series

$$
r(t)=\sum_{k=0}^{\infty} \rho_{k} t^{k}
$$

which converges absolutely for $|t|<1$.

Writing

$$
(1-t) w(t) r(t)=z^{2}[w(1)-w(t)]
$$

and using the series representation for $w(t)$, the coefficients for which can be found by the method of the last section are

$$
\left\{\omega_{0}+\sum_{k=1}^{\infty}\left(\omega_{k}-\omega_{k-1}\right) t^{k}\right\} \sum_{k=0}^{\infty} \rho_{k} t^{k}=z^{2}\left(\frac{2}{\pi}-\omega_{0}\right)-\sum_{k=1}^{\infty} z^{2} \omega_{k} t^{k}
$$


Multiplying the series on the left,

(5-15) $\sum_{k=0}^{\infty}\left\{\omega_{0} \rho_{k}+\sum_{j=1}^{k}\left(\omega_{j} \omega_{j-1}\right) \rho_{k-j}\right\} t^{k}=z^{2}\left(\frac{2}{\pi}-\omega_{0}\right)-\sum_{k=1}^{\infty} z^{2} \omega_{k} t^{k}$

It follows that the $\rho_{k}$ may be evaluated by solving the system of linear equations

$$
\rho_{0}=\frac{z}{\omega_{0}}\left(\frac{2}{\pi}-\omega_{0}\right)
$$

and, for $k \geqslant 1$,

$$
\rho_{k}=-\left\{z^{2} \frac{\omega_{k}}{\omega_{0}}+\sum_{j=1}^{k}\left(\frac{\omega_{j}}{\omega_{0}}-\frac{\omega_{j-1}}{\omega_{0}}\right) \rho_{k-j}\right\}
$$

The general solution of $(5-10)$ is

$$
d(t)=C / \sqrt{1-t}+d^{\dagger}(t)
$$

where $d^{\dagger}(t)$ may be taken as the solution giving the phase shift.

Setting

$$
d(t)=\sum_{k=0}^{\infty} \Delta_{k} t^{k}
$$

we have, in the domain of convergence,

$$
\sum_{k=0}^{\infty}\left[2(k+1) \Delta_{k+1}-(2 k+1) \Delta_{k}\right] t^{k}=\sum_{k=0}^{\infty} \rho_{k} t^{k}
$$

so that the $\Delta_{k}$ must satisfy the inhomogeneous recurrence

$$
2(k+1) \Delta_{k+1}-(2 k+1) \Delta_{k}=\rho_{k} \quad(k=0,1,2, \ldots) .
$$

We can solve this equation explicitly. The complementary solution is

$$
\bar{\Delta}_{k}=\frac{2 k-1}{2 k} \bar{\Delta}_{k-1}=\frac{(2 k) !}{\left(2^{k} k !\right)^{2}} \bar{\Delta}_{0}=(-1)^{k}\left(\begin{array}{c}
-1 / 2 \\
k
\end{array}\right) \bar{\Delta}_{0} .
$$

It follows that a solution to the equation

$$
2(k+1) \Delta_{k+1}^{n}-(2 k+1) \Delta_{k}^{n}=\rho_{n} \delta_{k, n}
$$

with $\delta_{k, n}$ the Kronecker $\delta$ symbol, is

$$
\Delta_{k}^{n}= \begin{cases}-\frac{(2 k) !}{\left(2^{k} k !\right)^{2}} \frac{\left(2^{n} n !\right)^{2}}{(2 n+1) !} \rho_{n}, & k \leqslant n, \\ 0, & k>n .\end{cases}
$$


Summing over $n$ gives us the general solution to (5.21)

$$
\Delta_{k}=\bar{\Delta}_{k}+\sum_{n=0}^{\infty} \Delta_{k}^{n}=\frac{(2 k) !}{\left(2^{k} k !\right)^{2}}\left\{\bar{\Delta}_{0}-\sum_{n=k}^{\infty} \frac{\left(2^{n} n !\right)^{2}}{(2 n+1) !} \rho_{n}\right\}
$$

Since $\left(2^{n} n !\right)^{2} /(2 n+1)$ ! is a monotone decreasing function of $n$, Dirichlet's test [10, p. 17] insures that $\Sigma\left(2^{n} n !\right)^{2} \rho_{n} /(2 n+1)$ ! converges whenever $\Sigma \rho_{n}$ does, and the sum from $k$ on must approach zero. It follows that

$$
\Delta_{k}^{\dagger}=-\frac{(2 k) !}{\left(2^{k} k !\right)^{2}} \sum_{n=k}^{\infty} \frac{\left(2^{n} n !\right)^{2}}{(2 n+1) !} \rho_{n}
$$

is a minimal solution to (5-21) and may be computed stably by backward recurrence once the $\rho_{k}$ have been found. The identification of $\left\{\Delta_{k}^{\dagger}\right\}$ with the power series coefficients for $d^{\dagger}(t)$ may be made by observing that $\lim _{t \rightarrow 1} d^{\dagger}(t)=\left(4 \nu^{2}-1\right) / 8$, while the complementary function is unbounded as $t \rightarrow 1-$.

Evaluation of the phase shift, $d^{\dagger}(t)$ thus consists of three principal tasks:

a. Computing the coefficients $\left\{\omega_{k}^{\dagger}\right\}$ for the given $\nu$ and $z$ using the backward recurrence of Section 4.

b. Finding the coefficients $\left\{\rho_{k}\right\}$ by solving the triangular system of linear equations (5-16) and (5-17).

c. Evaluating $\left\{\Delta_{k}^{\dagger}\right\}$ by backward application of (5-21), and summing the series (5-19) for the desired value (or values) of $t$, with $|t|<1$.

The procedure was tested numerically for the same values of $x, z$, and $\nu$ used for the recurrence of Section 4. Again, the results were in satisfactory agreement with tabulated values of the Bessel functions. Typical precision indexes obtained are presented in Table 5-1. The results support the following generalizations:

a. Convergence is rapid for large $|z|$, but the rate decreases with $|z|$. The choice $z=\sqrt{2 x}$ gives more rapid convergence than $z=x$.

b. The precision index may be approximated by an expression of the form (3-29), although the approximation is not so good as for $x\left|H_{\nu}^{(1)}(x)\right|^{2}$ or for $e^{x} K_{\nu}(x)$. The constant $\alpha$ decreases from 2.6 to 0.5 and $\beta$ from 0.73 to 0.61 as $z$ decreases from $16 \sqrt{2}$ to 2 .

c. The precisions for the phase shifts are about three digits less than for the corresponding squared moduli. It should be observed, however, that the phase shift is a relatively small correction to the asymptotic phase, $\chi$.

d. Precisions are almost independent of $\nu$ for $\nu\langle|z|$. For $\nu>|z|$, the precision may deteriorate rapidly because of the complex zeros of $H_{\nu}^{(1)}(x)$ in the right half plane.

e. The results appear to be sensitive to the arithmetic precision used. For long precision IBM System 370 arithmetic (14 hexadecimal, approximately 16 decimal, digit fraction) random fluctuations in precision index were observed when the precision index approached about 11.5. Carrying out the calculations in extended precision (about 35S) arithmetic eliminated the fluctuations. 


\section{TABLE 5-1}

Precision indexes for Bessel function phase shifts

$\begin{array}{cccccccccr}x & 2 & 2 & 4 & 4 & 4 & 4 & 8 & 8 & 16 \\ 2 & 2 & 2 \sqrt{2} & 4 & 4 & 4 & 4 & 8 & 8 \sqrt{2} & 16 \\ v & 0 & 0 & 0 & 1 & 4 & 5 & 0 & 0 & 0 \\ N & & & & & \text { Indexes of Precision }\end{array}$

$\begin{array}{lllllrrrrr}1 & 0.39 & 0.65 & 0.83 & 1.05 & -1.06 & 0.80 & 1.38 & 1.76 & 1.96 \\ 2 & 0.70 & 1.10 & 1.43 & 1.71 & -0.29 & -0.51 & 2.42 & 3.07 & 3.56 \\ 3 & 0.95 & 1.47 & 1.91 & 2.23 & 0.61 & -1.39 & 3.26 & 4.16 & 4.89 \\ 4 & 1.18 & 1.77 & 2.31 & 2.65 & 1.86 & -0.00 & 3.97 & 5.08 & 6.05 \\ 5 & 1.37 & 2.04 & 2.66 & 3.02 & 2.37 & 0.92 & 4.58 & 5.88 & 7.08\end{array}$

$\begin{array}{rrrrrrrrrr}6 & 1.55 & 2.28 & 2.98 & 3.34 & 2.51 & 2.20 & 5.13 & 6.60 & 7.99 \\ 7 & 1.71 & 2.50 & 3.27 & 3.64 & 2.89 & 1.52 & 5.63 & 7.25 & 8.82 \\ 8 & 1.87 & 2.71 & 3.53 & 3.90 & 3.86 & 1.66 & 6.08 & 7.84 & 9.59 \\ 9 & 2.01 & 2.89 & 3.77 & 4.15 & 3.99 & 2.68 & 6.49 & 8.38 & 10.29 \\ 10 & 2.14 & 3.07 & 4.00 & 4.39 & 5.07 & 1.80 & 6.88 & 8.89 & 10.95\end{array}$

$\begin{array}{rrrrrrrrrr}11 & 2.26 & 3.23 & 4.21 & 4.60 & 4.29 & 1.92 & 7.25 & 9.37 & 11.57 \\ 12 & 2.38 & 3.39 & 4.42 & 4.81 & 4.41 & 3.54 & 7.59 & 9.82 & 12.16 \\ 13 & 2.49 & 3.54 & 4.61 & 5.00 & 4.92 & 2.14 & 7.92 & 10.24 & 12.74 \\ 14 & 2.60 & 3.68 & 4.79 & 5.19 & 6.11 & 2.21 & 8.23 & 10.65 & 13.39 \\ 15 & 2.70 & 3.81 & 4.97 & 5.37 & 6.25 & 3.22 & 8.52 & 11.04 & \end{array}$

$\begin{array}{lllllllll}16 & 2.80 & 3.94 & 5.13 & 5.54 & 5.71 & 2.46 & 8.80 & 11.42 \\ 17 & 2.90 & 4.07 & 5.30 & 5.70 & 5.73 & 2.48 & 9.07 & 11.80 \\ 18 & 2.99 & 4.19 & 5.45 & 5.86 & 5.98 & 3.25 & 9.34 & 12.20 \\ 19 & 3.08 & 4.30 & 5.60 & 6.01 & 6.31 & 2.79 & 9.59 & 12.70 \\ 20 & 3.17 & 4.42 & 5.75 & 6.15 & 6.54 & 2.76 & 9.83 & \end{array}$

$\begin{array}{llllllll}21 & 3.25 & 4.52 & 5.89 & 6.30 & 6.63 & 3.38 & 10.06 \\ 22 & 3.33 & 4.63 & 6.02 & 6.43 & 6.73 & 3.12 & 10.29 \\ 23 & 3.41 & 4.73 & 6.15 & 6.57 & 6.88 & 3.04 & 10.51 \\ 24 & 3.49 & 4.83 & 6.28 & 6.70 & 7.05 & 3.55 & 10.73 \\ 25 & 3.56 & 4.93 & 6.41 & 6.82 & 7.21 & 3.46 & 10.94\end{array}$

As suggested by $\mathrm{d}$ above, the domain of applicability of this procedure is limited by the requirement that the series for $r(t)$ converge for $|t|<1$. This demands not only that $w(t)$ have no singularities other than poles in the unit disc, but also that it not vanish there. Although $H_{\nu}^{(1)}(x)$ has no real zeros, it does have complex zeros, some of which lie in the right half plane.

To find the restriction on permissible values of $z^{2}$ imposed by a particular zero $x_{j}$, we may let

$$
z^{2}=\rho e^{i \theta} x_{j}^{2}
$$

so that the condition $\left|t\left(x_{j}\right)\right|^{2}>1$ becomes

$$
\left|1-\rho e^{i \theta}\right|^{2}=1-2 \rho \cos \theta+\rho^{2}>1
$$


or

$$
\rho>2 \cos \theta .
$$

In particular, using Döring's [11] values for the zeros of $H_{n}^{(1)}(x)$, and limiting our attention to real $z$, we find that for $\nu=4$, we must have $z>1.376 \ldots$, while for $\nu=5$, we must have $z>3.088$. . . These limits may be confirmed by attempting to perform the algorithm for smaller values of $z$. It will be found that $\Sigma \rho_{k}$ diverges badly, so that backward recurrence for the $\Delta_{k}^{\dagger}$ fails.

6. Discussion and Conclusions. Of the four recurrences presented, the first is useful for small arguments, while the others are most effective near $\infty$. The first, Eq. (2-9), is competitive with other methods of evaluating $J_{\nu}(x)$ but offers no clear advantage over the power series for $|x|$ less than the first zero. This should not be surprising in view of the close connection between recurrences and continued fractions, and the observation that continued fractions corresponding to power series for entire functions rarely accelerate convergence, although those corresponding to asymptotic divergent series often show dramatic improvement.

The last three recurrences fall in the latter class, and not only converge more rapidly, but also yield higher precisions than the asymptotic series over wider domains of the complex argument. Hitotumatu's continued fraction, Eq. (3-21), shows comparable convergence but requires a more complicated normalization. It, thus, appears that the recurrences should replace the asymptotic series for large arguments and relatively small orders. Equation (3-11) with $z=-i x$ provides an improvement over the Hankel form, although the modulus and phase representation deserves careful consideration.

Acknowledgments. The programming assistance of Ms. Jyawei K. Wu and of Dr. Gregory Hively is gratefully acknowledged. Dr. Hively also contributed significantly to the clarity and correctness of the analytical development.

Department of Computer Science

University of Kentucky

Lexington, Kentucky 40506

1. J. C. P. MILLER, British Association for the Advancement of Science Mathematical Tables. Vol. X. Bessel Functions. Part II, Functions of Positive Integer Order, Cambridge University Press, Cambridge, 1952.

2. W. GAUTSCHI, "Computational aspects of three-term recurrence relations," SIAM Rev., v. 9, 1967 , pp. 24-82.

3. W. GAUTSCHI, “Zur Numerik rekurrenter Relationen,” Computing, v. 9, 1972, pp. 107126.

4. W. GAUTSCHI, "Computational methods in special functions-a survey," Theory and Applications of Special Functions, R. Askey, (ed.), Academic Press, New York, 1975, pp. 1 -98.

5. H. C. THACHER, JR., "Series solutions to differential equations by backward recurrence," Information Processing 71, North-Holland, Amsterdam, 1972, pp. 1287-1291.

6. F. W. J. OLVER, "9. Bessel functions of integer order," Handbook of Mathematical Functions with Formulas, Graphs, and Mathematical Tables, M. Abramowitz and I. A. Stegun, (eds.), Nat. Bur. Standards Appl. Math. Series 55, U. S. Government Printing Office, Washington, D. C., 1964 , pp. $355-433$. 
7. H. A. ANTOSIEWICZ, “10. Bessel functions of fractional order," Handbook of Mathematical Functions with Formulas, Graphs and Mathematical Tables, M. Abramowitz and I. A. Stegun, (eds.), Nat. Bur. Standards Appl. Math. Series 55, U. S. Government Printing Office, Washington, D. C., 1964, pp. 435-478.

8. F. W. J. OLVER, Asymptotics and Special Functions, Academic Press, New York, 1974.

9. S. HITOTUMATU, "On the numerical computation of Bessel functions through continued fractions," Comment. Math. Univ. St. Paul, v. 16, 1967/68, pp. 89-113.

10. E. T. WHITTAKER \& G. N. WATSON, A Course of Modern Analysis, 4th ed., Cambridge University Press, Cambridge, 1927.

11. B. DÖRING, "Complex zeros of cylinder functions," Math. Comp., v. 20, 1966, pp. 215-222. 